\title{
ESTUDO CINÉTICO DA DEGRADAÇÃO HETEROGÊNEA DO FENANTRENO UTILIZANDO PERSULFATO DE SÓDIO E DIATOMITA MODIFICADA
}

\author{
C. K. O. Silva; L. G. Aguiar; M. M. G. R. Vianna; C. A. O. Nascimento.
}

Departamento de Engenharia Química, Universidade de São Paulo, São Paulo-SP

celyna@usp.br

\begin{abstract}
RESUMO - A remediação de solo e sedimentos contaminados com hidrocarbonetos policíclicos aromáticos, ainda é um desafio científico e técnico, pois estes compostos apresentam elevada estabilidade e alta afinidade biológica. $\mathrm{O}$ objetivo deste trabalho foi avaliar a viabilidade de utilização de oxidação química com persulfato de sódio como oxidante para degradar o fenantreno, utilizando como catalisador um material ativador heterogêneo, a base de diatomita (suporte) e óxidos/hidróxidos de ferro (fase ativa). Para isto, testes em escala de bancada foram realizados para tratar areia contaminada com $200 \mathrm{mg}$ de fenantreno por $\mathrm{kg}$ de solo. Resultados satisfatórios foram obtidos em 7 dias de tratamento, tendo como contaminação residual $36 \mathrm{mg} / \mathrm{kg}$, valor abaixo do limite da intervenção holandesa. Foi realizada também, a modelagem matemática da degração do fenantreno, com base no mecanismo LHHW, considerando a dessorção como etapa lenta. $\mathrm{O}$ modelo explicou satisfatoriamente os experimentos, com um coeficiente $\mathrm{R}^{2}>0,9$.
\end{abstract}

\section{INTRODUÇÃO}

A agência ambiental americana United States Environmental Protection Agency (USEPA) publicou uma lista com 126 poluentes prioritários. Fazem parte dessa lista 16 hidrocarbonetos policiclicos aromáticos (HPA), entre eles o fenantreno. Dentre os critérios para os poluentes pertencerem a essa lista, podem ser citados: apresentarem toxicidade ao homem e ao meio ambiente e serem persistentes e biocumulativos no meio ambiente. A USEPA regulamenta e dispõe de métodos analíticos para identificar e quantificar esses poluentes (EPA, 2012).

De acordo com a regulamentação holandela, norma internacional para valores de intenvenção de contaminantes no meio ambiente subterrâneo, o limite de intervenção para solo contaminado com HPA é de $40 \mathrm{mg}$ por $\mathrm{kg}$ de solo seco. Esse valor indica um nível de qualidade do solo no qual existem riscos para a saúde humana e para o ambiente. Portanto, em um solo com teores de HPA acima de $40 \mathrm{mg} / \mathrm{kg}$, é necessária a implementação de ações voltadas para a sua remediação.

Processos de tratamento de solo contaminados com HPA por incineração e escavação são limitados e apresentam custo elevado. Os tratamentos do solo in situ, com estes compostos, são frequentemente mais utilizados por apresentarem melhor custo-benefício do que a remediação ex situ, (Palmroth et al, 2006). 
Dentre as técnicas de tratamento in situ, tem-se a oxidação química in situ (In Situ Chemical Oxidation - ISCO), que é uma técnica viável para a remediação de aquíferos, sedimentos e solos contaminados com poluentes orgânicos. ISCO envolve a introdução de um oxidante químico no subsolo com a finalidade de transformar os contaminantes nas águas subterrâneas e/ou no solo em espécies químicas menos nocivas, podendo estas espécies chegar a mineralização completa (EPA, 2006).

$\mathrm{O}$ persulfato $\left(\mathrm{S}_{2} \mathrm{O}_{8}{ }^{-2}\right)$ é uma forma relativamente nova de oxidante que tem sido investigada. A oxidação por persulfato tem sido aplicada para degradar vários contaminantes, como eteno clorados, etano clorados, clorofenóis, bisfenol, HPA, PCB, componentes e aditivos de gasolina, e ainda vários compostos orgânicos voláteis (Gao et al, 2012; Usman et al, 2012; Nfodzo et al, 2011). A capacidade do persulfato de gerar radicais livres é particularmente importante para aplicações de remediação. (Tsitonaki et al, 2010).

O persulfato pode ser ativado por diferentes métodos para formar o poderoso radical sulfato $\left(\mathrm{SO}_{4}-\bullet\right)$, esse radical tem potencial redox de $2,6 \mathrm{~V}$, portanto mais alto do que o potencial do íon persulfato $(2,1 \mathrm{~V})$, desse modo a reatividade do radical será maior. Os métodos de ativação desse oxidante podem ser: luz UV, calor, metal de transição, meio alcalino, peróxido de hidrogênio e ozônio.

Com a ativação por metais de transição (representado por $\mathrm{M}$ ) um mol de persulfato produz 1 mol de radical, equação (1).

$$
\mathrm{S}_{2} \mathrm{O}_{8}{ }^{2-}+\mathrm{M} \rightarrow \mathrm{M}^{+}+\mathrm{SO}_{4}{ }^{2-}+\mathrm{SO}_{4}{ }^{-}
$$

O método de ativação proposto neste trabalho trata-se de um catalisador heterogêneo constituído por um material suporte (diatomita) sobre o qual é precipitada uma mistura de íons ferroso $\left(\mathrm{Fe}^{+2}\right)$ e férrico $\left(\mathrm{Fe}^{+3}\right)$ na forma de óxidos e hidróxidos de ferro (Figura 1).

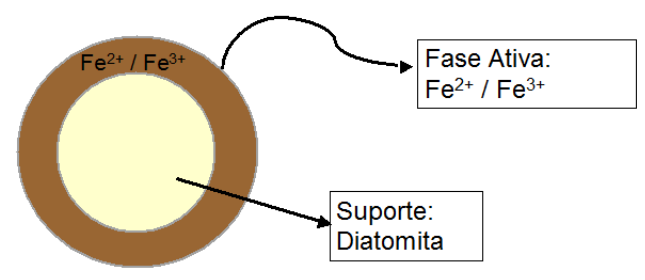

Figura 1: Esquema da partícula da diatomita modificada

Dentre os tipos de catalisadores, o mais utilizado é constituído por duas fases, uma suporte e a outra o material ativo, obtido por impregnação, precipitação ou precipitação por deposição. Em geral, o suporte não é uma fase ativa, mas serve para aumentar a área e dispersar a fase ativa. A fase ativa pode ser um metal ou óxido, que é o componente ativo para interagir com as moléculas durante a reação química (Schmal, 2011).

A diatomita é composta pela sílica $\left(\mathrm{SiO}_{2}\right)$ e diversas impurezas, como certos minerais e produtos químicos (especialmente sob a forma de ferro). (Jia et al, 2007; Zhaolun et al, 2005). A elevada porosidade da diatomita a torna adequada para a modificação da superfície, 
podendo ser um material de suporte apropriado em alguns casos, utilizando-se a modificação da diatomita bruta. (Xiong \& Peng, 2008).

Neste trabalho, os objetivos foram preparar um material heterogêneo, com a diatomita como suporte, para ativar o persulfato de sódio para o tratamento de HPA e estudar a cinética da degradação do fenantreno em sistema heterogêneo. Foi estudada a degradação desse contaminante, porque ele é considerado um contaminante modelo em estudos que envolvem a interação de HPA com o meio ambiente. Foi avaliada a degradação do fenantreno em matriz sólida, utilizando areia como solo modelo. A areia foi selecionada para evitar uma possível competição que pode ocorrer entre os materiais de interferência do solo real e o oxidante. Dentre as interferências, podem ser consideradas: matéria orgânica, metais e sorção de contaminantes que podem ocorrer em matrizes argilosas.

\section{METODOLOGIA}

\subsection{Síntese e caracterização do material}

A modificação da diatomita foi realizada utilizando-se $100 \mathrm{~g}$ de diatomita bruta, na qual foi adicionada lentamente a $112 \mathrm{~mL}$ de uma solução aquosa contendo: $\mathrm{Fe}_{2}\left(\mathrm{SO}_{4}\right)_{3}$ (366 g. $\left.\mathrm{L}^{-1}\right)$ e $\mathrm{FeSO}_{4} .7 \mathrm{H}_{2} \mathrm{O}\left(183 \mathrm{~g} . \mathrm{L}^{-1}\right)$, em um reator de mistura intensa. Nessa suspensão foram adicionadas, em 4 etapas, $220 \mathrm{~mL}$ de uma solução de $\mathrm{NaOH}$ a $5 \mathrm{~mol} \cdot \mathrm{L}^{-1}$. A mistura permaneceu em homogeneização por 1 hora. $\mathrm{O}$ material sintetizado foi lavado por filtração a vácuo, para remoção dos possíveis subprodutos indesejáveis formados durante a síntese e do excesso de $\mathrm{NaOH}$. Após a lavagem, o material foi mantido em estufa a $60^{\circ} \mathrm{C}$ por 24 horas. Após a secagem, o catalisador foi moído (desagregado), com almofariz e pistilo e depois peneirado e condicionado em frascos de vidro.

Para caracterizar o material foram realizadas análises de difração e fluorescência de raio-x nas amostras de diatomita bruta e diatomita modificada.

\subsection{Experimentos em batelada}

Em cada erlenmeyer (reator) de $125 \mathrm{~mL}$, foram pesados 15 gramas da areia contaminada com $200 \mathrm{mg}$ de fenantreno, sendo adicionados os volumes das soluções do oxidante e completados com água mili-Q até o volume de $30 \mathrm{~mL}$. Para as reações com o catalisador, a quantidade de catalisador foi adicionada no frasco e este foi homogeneizado. Os experimentos foram realizados em duplicatas para se avaliar dos erros. Os erros experimentais neste trabalho foram abaixo de $5 \%$.

Os frascos foram condicionados em banho termostático a $32^{\circ} \mathrm{C}$. Ensaios controles (sem adição de oxidante) foram realizados para cada reação, nas mesmas condições de tratamento.

Foram realizados testes com persulfato de sódio ativado pelo método convencional via ferro e comparados com a ativação promovida pela diatomita modificada. O tempo de remediação para esse teste foi mantido constante em 72 horas de tratamento. As condições do oxidante, $\mathrm{Fe}^{2+}$ e quantidade de diatomita e pH estão apresentadas na Tabela 1. 
Tabela 1: Condições experimentais

\begin{tabular}{c|c|c|c|c}
\hline Oxidantes & $\begin{array}{c}\text { Concentração } \\
\text { Oxidante } \\
\left(\mathrm{mol.L}^{-1}\right)\end{array}$ & $\begin{array}{c}\text { Concentração } \\
\text { Sulfato Ferroso } \\
\left(\mathrm{mol.L}^{-1}\right)\end{array}$ & $\begin{array}{c}\text { Catalisador } \\
\text { (gramas)* }\end{array}$ & $\mathrm{pH}_{\text {inicial }}$ \\
\hline $\begin{array}{c}\text { Persulfato de sódio } \\
\text { Tradicional } \\
\text { (PS Tradicional) }\end{array}$ & 0,1 & 0,03 & 3 \\
\hline $\begin{array}{c}\text { Persulfato de sódio } \\
+ \text { diatomita } \\
\text { modificada } \\
\text { (PS+MD) }\end{array}$ & 0,1 & & 2,5 & 11 \\
\hline
\end{tabular}

Para estudo da taxa da reação em catálise heterogênea foi utilizado o persulfato de sódio a concentração 0,1 mol. $\mathrm{L}^{-1}$ e $2,5 \mathrm{~g}$ de diatomita modificada nos tempos 4h, 24h, 48h, $72 \mathrm{~h}$ e $168 \mathrm{~h}$ de tratamento e em $\mathrm{pH} 11$.

Para a degradação do fenantreno utilizando o oxidante persulfato de sódio, foi considerada a equação (2).

$$
33 \mathrm{Na}_{2} \mathrm{~S}_{2} \mathrm{O}_{8}+\mathrm{C}_{14} \mathrm{H}_{10}+28 \mathrm{H}_{2} \mathrm{O} \rightarrow 14 \mathrm{CO}_{2}+66 \mathrm{Na}^{+}+66 \mathrm{HSO}_{4}^{-}
$$

\subsection{Extração e Análise}

Para quantificação da contaminação residual e dos possíveis sub-produtos formados durante a oxidação, alíquotas de 2,5 gramas de areia foram retiradas de cada reator, sendo cada alíquota adicionada em vial de $40 \mathrm{~mL}$, juntamente com $10 \mathrm{~mL}$ de solvente hexano:cetona (1:1). A homogeneização, amostra-solvente, foi realizada utilizando vortex por 5 segundos. Em seguida, cada vial com amostra e solvente foi submetido a ultrassom durante 30 minutos para extração sólido-liquido. Após a extração, cada amostra foi passada pelo vortex novamente por 5 segundos. $O$ extrato foi filtrado, por filtração comum, contendo sulfato de sódio no papel de filtro. Esse extrato filtrado foi então colocado em vial de $1,5 \mathrm{~mL}$ para injeção em um Cromatógrafo Gasoso acoplado ao Espectrômetro de Massa (CG-EM), modelo GC-17A da Shimadzu. Os métodos analíticos, extração e análise, foram empregados de acordo com métodos USEPA.

\subsection{Modelagem matemática}

O processo de degradação foi estudado através do mecanismo LangmuirHinshelwood-Hougen-Watson (LHHW) de reação heterogênea. No presente estudo, foram consideradas as etapas de adsorção dos reagentes (Fenantreno e Persulfato de sódio), reação superficial na diatomita modificada e dessorção dos produtos. A equação (3) mostra a taxa global de degradação considerando a dessorção como etapa lenta. 


$$
R_{\text {Global }}=\frac{k_{D} C_{t}\left(K_{R S} K_{O} K_{F} C_{O} C_{F}-\frac{C_{P}}{K_{D}}\right)}{\left(1-K_{O} C_{O}+K_{F} C_{F}+K_{O} K_{F} C_{O} C_{F}\right)}
$$

sendo que:

$\mathrm{k}_{\mathrm{D}}$ : Constante cinética de dessorção de produtos $\left(\mathrm{h}^{-1}\right)$

$\mathrm{K}_{\mathrm{D}}$ : Constante de equilíbrio dessorção / adsorção de produtos

$\mathrm{K}_{\mathrm{F}}$ : Constante de equilíbrio adsorção / dessorção de fenantreno

$\mathrm{K}_{\mathrm{O}}$ : Constante de equilíbrio adsorção / dessorção de oxidante

$\mathrm{K}_{\mathrm{RS}}$ : Constante de equilíbrio químico da reação superficial

$\mathrm{C}_{\mathrm{F}}$ : Concentração de fenantreno $\left(\mathrm{mol} . \mathrm{L}^{-1}\right)$

$\mathrm{C}_{\mathrm{O}}$ : Concentração de oxidante $\left(\mathrm{mol} . \mathrm{L}^{-1}\right)$

$\mathrm{C}_{\mathrm{t}}$ : Concentração total de sítios no sólido (mol. $\left.\mathrm{L}^{-1}\right)$

\section{RESULTADOS}

\subsection{Caracterização do material}

De acordo com a análise de fluorescência de raio-x, os teores de íons de ferro total foram 4,78\%, 17,65\%, para as amostras diatomita bruta e diatomita modificada, respectivamente. Esses resultados indicam significativo aumento na quantidade de íons de ferro após a síntese.

Nos resultados da análise de difração de raio-x, não foi possível detectar a presença dos óxidos ou hidróxidos de ferro formados, pois o material formou cristais em quantidades muito baixas para serem detectados, implicando em característica amorfa das diatomitas bruta e modificada.

\subsection{Experimentos em batelada}

A partir dos resultados apresentados na Figura 2, verifica-se que o teste utilizando o oxidante persulfato de sódio com diatomita modificada obteve melhor resultado do que o obtido pelo de método de ativação convencional, utilizando sulfato ferroso. Os percentuais de redução do fenantreno para a ativação convencional e pela diatomita modificada foram, $20 \%$ e $56 \%$ respectivamente.

De acordo com o resultado do ensaio Controle DM, que trata-se do ensaio com catalisador sem adição de oxidante, observa-se que o material sintetizado apresenta 
propriedade de adsorção do fenantreno, uma vez que houve redução da concentração desse contaminante em $15 \%$ nesse ensaio.

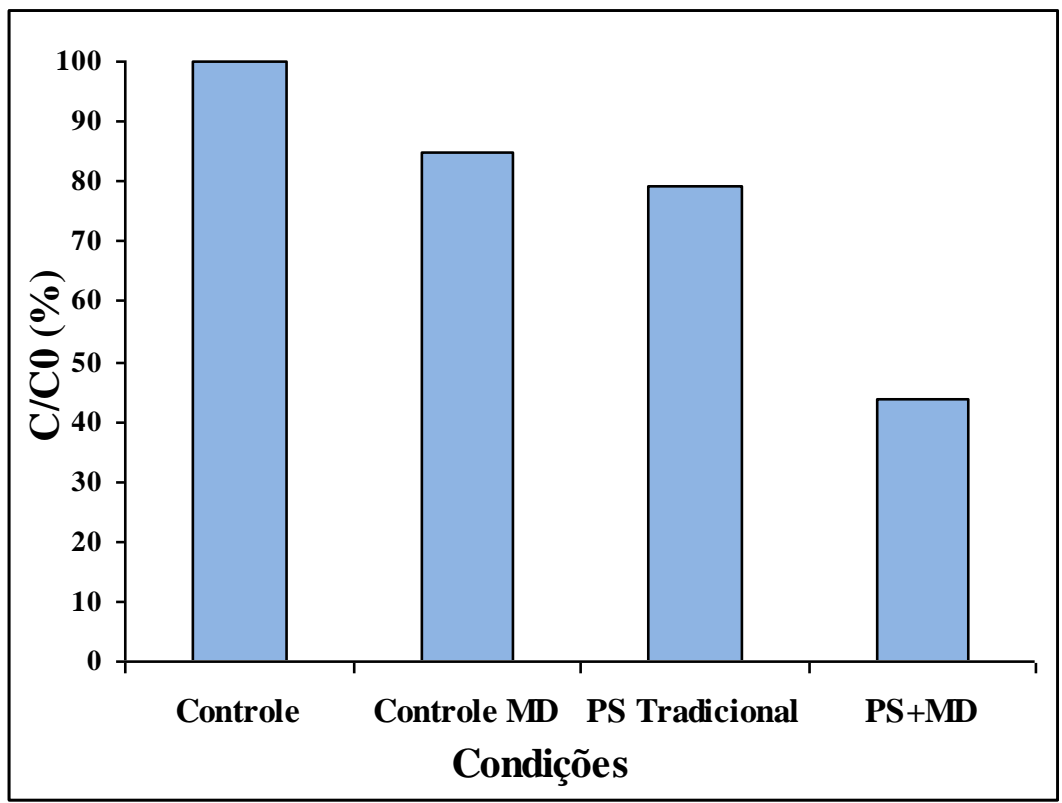

Figura 2: Comparação de métodos de ativação do persulfato de sódio com método convencional de ativação (PS Tradicional) e com a diatomita modificada (PS+MD). Persulfato de sódio a 0,1 mol.L $\mathrm{L}^{-1}$ $\left(25\right.$ g. $\left.\mathrm{L}^{-1}\right)$ e $2,5 \mathrm{~g}$ de catalisador, 72 horas de remediação e temperatura de $32^{\circ} \mathrm{C}$.

$\mathrm{Na}$ análise da degradação do fenantreno não foi detectado intermediário. Portanto, para a modelagem cinética heterogênea foi considerada que a porcentagem de fenantreno degradada refere-se ao teor de mineralização do mesmo.

De acordo com a Figura 3, resultados satisfatórios foram obtidos em 7 dias de tratamento, tendo como contaminação residual $36 \mathrm{mg}$ de fenantreno por $\mathrm{kg}$ de solo, valor abaixo do limite da intervenção holandesa para teores totais de HPA no solo.

O modelo matemático aqui desenvolvido explicou adequadamente os experimentos, com um coeficiente de correlação $\mathrm{R}^{2}>0,9$ (Figura 4).

Na simulação foram utilizados os seguintes dados:

$$
\mathrm{k}_{\mathrm{d}}=0,84 \mathrm{~h}^{-1} ; \mathrm{K}_{\mathrm{O}}=0,89 ; \mathrm{K}_{\mathrm{F}}=0,84 ; \mathrm{K}_{\mathrm{D}}=1 ; \mathrm{K}_{\mathrm{RS}}=0,84 \text { e } \mathrm{C}_{\mathrm{t}}=0,2 \mathrm{~mol} / \mathrm{L} \text {. }
$$




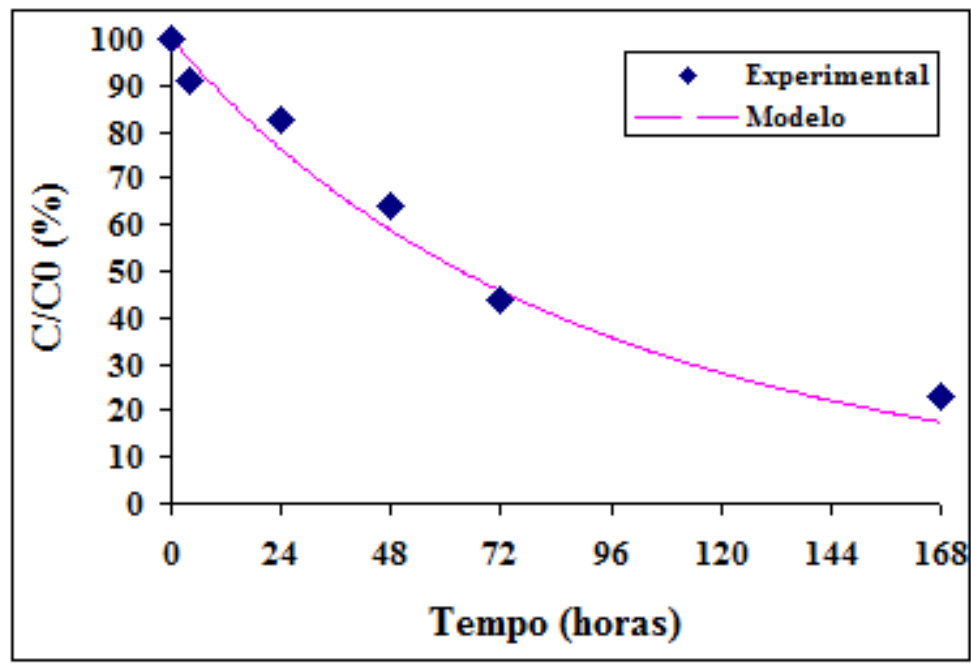

Figura 3: Degradação de fenantreno em função do tempo. Persulfato de sódio a 0,1 mol. $\mathrm{L}^{-1}$ e 2,5g de diatomita modificada e temperatura de $32^{\circ} \mathrm{C}$.

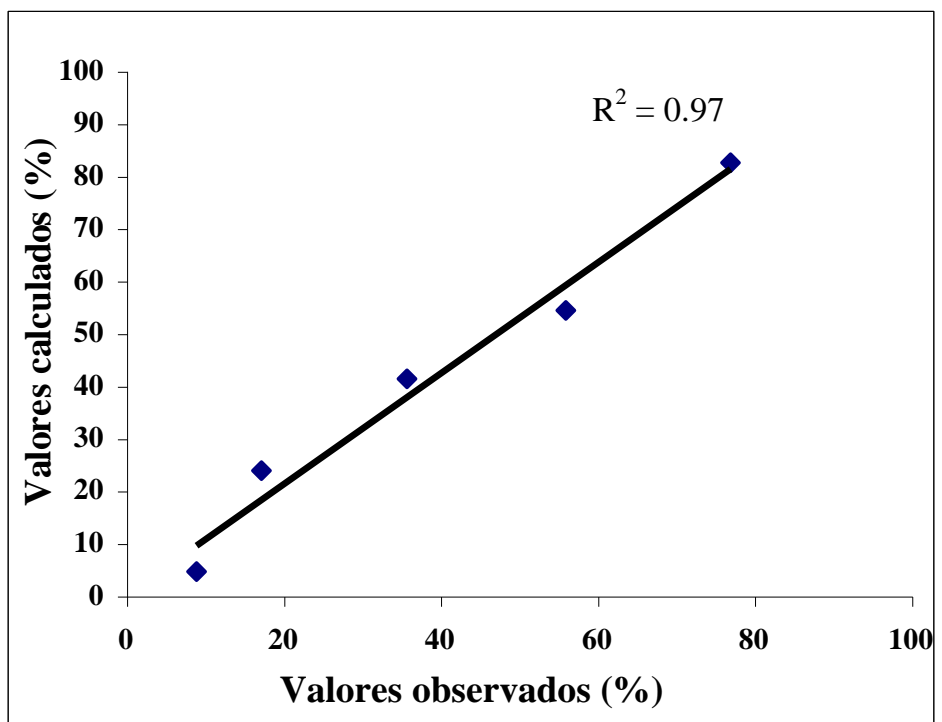

Figura 4: Valores de degradação do fenantreno observados experimentalmente versus valores calculados

\section{CONCLUSÕES}

A partir dos resultados da avaliação da eficiencia do catalisador, pode-se concluir que o material sintetizado apresenta significativa atividade catalítica para oxidar o fenantreno, quando utilizado em conjunto com o persulfato de sódio como oxidante. Também foi observado que uma parcela da remoção do contaminante deve-se à propriedade de adsorção do contaminante na diatomita modificada. Resultados satisfatórios foram obtidos utilizando $2,5 \mathrm{~g}$ de diatomita modificada com persulfato de sódio a $0,1 \mathrm{~mol} \cdot \mathrm{L}^{-1}$, durante o tempo de remediação de 7 dias, onde foram obtidos contaminantes residuais de $36 \mathrm{mg}$ de fenantreno por 
$\mathrm{kg}$ de solo seco, valores esses abaixo dos limites da intervenção holandesa. Na modelagem cinética proposta neste trabalho, considerando a dessorção dos produtos como a etapa lenta, o mecanismo proposto explicou bem o processo com coeficiente de correlação $\mathrm{R}^{2}>0,9$.

\section{REFERÊNCIAS}

EPA, 2012. Toxic and Priority Pollutants. Disponível em:

<http://water.epa.gov/scitech/methods/cwa/pollutants-background.cfm>. Acesso em: 17 de abril 2013.

EPA, 2006. Chemical Oxidation. Disponível em:<http://www.epa.gov/swerust1/pubs/tum_ch13.pdf>. Acesso em: 11 de abril 2013.

GAO, Y.; GAO, N.; DENG, Y.; YANGA; YI-QIONG; MAA; Yan. Ultraviolet (UV) lightactivated persulfate oxidation of sulfamethazine in water. Chemical Engineering Journal. 195-196 (2012) 248-253.

JIA, Y.; HANA, W.; XIONGA. G.; YANGA, W. Diatomite as high performance and environmental friendly catalysts for phenol hydroxylation with $\mathrm{H} 2 \mathrm{O} 2$. Science and Technology of Advanced Materials. 8 (2007) 106-109.

NFODZO, P.; CHOI, H. Sulfate Radicals Destroy Pharmaceuticals and Personal Care Products. Enviromental Engineering Science, Volume 28, Number, 8, 2011.

PALMROTH, M. R. T.; LANGWALDT, J.H.; AUNOLA, T.A.; GOI, A.; MÜNSTER, U. PUHAKKA, J.A.; TUHKANEN, T.A. Effect of modified Fenton's reaction on microbial activity and removal of PAHs in creosote oil contaminated soil. Biodegradation, 2006.

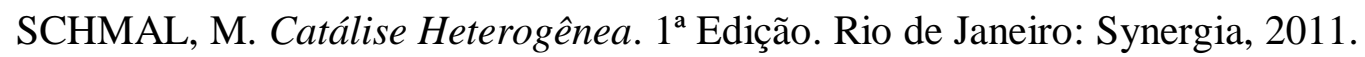

TSITONAKI, A.; PETRI, B.; CRIMI, M.; MOSBÆK, H.; SIEGRIST, R. L.; BJERG, P. L. In Situ Chemical Oxidation of Contaminated Soil and Groundwater Using Persulfate: A Review. 2010. Disponível em: http: http://www.tandfonline.com/doi/abs/10.1080/10643380802039303. Acesso em: 02 de abril, 2013.

USMAN, M; FAURA, P; HANNA, K. ABDELMOULA. M; RUBBY, C., Application of magnetite catalyzed chemical oxidation (Fenton-like and persulfate) for the remediation of oil hydrocarbon contamination. Fuel. $\underline{96}$, 270-276, 2012.

XIONG, W.; PENG. J. Development and characterization of ferrihydrite-modified diatomite as a phosphorus adsorbent. Water research. 42(2008)4869-4877.

ZHAOLUN, W.; YUXIANG, Y.; XUPING, Q.; JIANBO, Z.; YARU, C.; LINXI, N. Decolouring mechanism of Zhejiang diatomite. Application to printing and dyeing wastewater. Environ Chem Lett (2005) 3:33-37 Springer-Verlag 2005. 